Proceedings of the 45th International School and Conference on the Physics of Semiconductors "Jaszowiec" 2016, Szczyrk

\title{
Waiting Time Correlations in Transport through Two Coupled Quantum Dots
}

\author{
K. PTASZYŃSKI* \\ Institute of Molecular Physics, Polish Academy of Sciences, M. Smoluchowskiego 17, 60-179 Poznań, Poland
}

Waiting times between subsequent tunneling events in the double quantum dot system are shown to be correlated. The magnitude and the sign of the cross-correlation depend on the degree and the character of modulation of tunneling rates in the one dot due to the interaction with the charge state of the other, as well as on the relation between different time scales of the system dynamics.

DOI: 10.12693/APhysPolA.130.1233

PACS/topics: 72.70. $+\mathrm{m}, 73.23 . \mathrm{Hk}, 73.63 . \mathrm{Kv}$

\section{Introduction}

Current fluctuations can give useful information about the underlying mechanism of the mesoscopic transport. There are two approaches to the study of these fluctuations. Full counting statistics (FCS) analyses the number of transferred particles in the given time interval. In an alternative approach, distribution of time delays between subsequent physical events, known as waiting time distribution (WTD), is studied [1]. In quantum dots both FCS and WTD can be studied using charge detection techniques [2].

Dasenbrock et al. [3] have shown the correlation of subsequent waiting times in the coherent transport through a quantum point contact described within the scattering matrix formalism. Here the study is extended to the case of quantum dots. I show the presence of the waiting time correlation in the sequential tunneling transport through the capacitively interacting quantum dots, where the transport in one quantum dot is modulated by the charge state of the other. All calculations are made within the Markovian master equation formalism.

\section{System and methods}

I consider the four-terminal system of two capacitively coupled quantum dots (Fig. 1a). Similar system was studied previously both experimentally [4] and theoretically [5]. Each dot is coupled to different left (source) and right (drain) leads. The voltage bias is assumed to be high in comparison with the temperature, such that transport is unidirectional (the tunneling against the bias due to thermal excitations is not possible). I assume that tunneling rates between one dot and leads depend on the charge state of the other dot due to the energy dependence of the density of states in leads. It is possible experimentally to make tunneling rates energy dependent in a controllable manner, as shown recently by Thierschmann et al. [6]. Finally, I assume a strong intra-dot

*e-mail: krzysztof.ptaszynski@ifmpan.poznan.pl

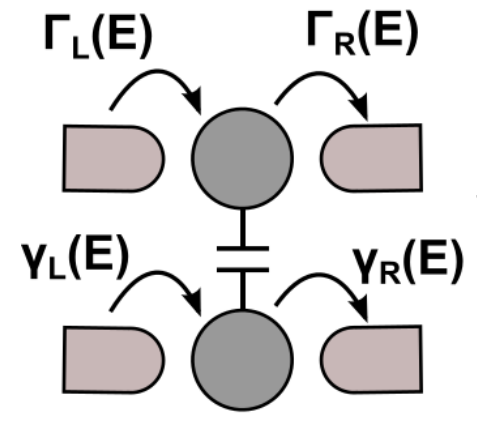

a)

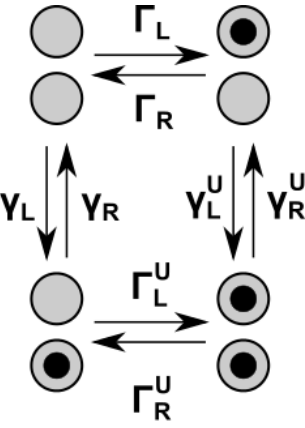

b)
Fig. 1. (a) System of two capacitively coupled quantum dots; $\Gamma_{i}(E)$ and $\gamma_{i}(E)$ - energy dependent tunneling rates. (b) Diagram of possible charge configurations with transition rates between them. Black dots denote electrons occupying quantum dots. Superscripts ${ }^{U}$ in the tunneling rates refers to the situation, when the other dot is charged.

Coulomb repulsion - each dot can be occupied by a maximally one electron. Thus, there are four possible charge configurations of the whole system, with corresponding transition rates between them (see Fig. 1b).

In order to study the transport in the double quantum dot system I use the Markovian master equation

$$
\dot{\rho}(t)=\mathcal{L} \rho(t),
$$

where $\rho(t)$ - a column vector containing state probabilities, $\mathcal{L}$ - a square matrix representing the Liouvillian. In the studied system coherences are assumed to be absent. I focus on the stationary state with $\dot{\rho}(t)=0$. The WTD is calculated using the approach developed by Brandes [1]. The Liouvillian is split in the following way:

$$
\mathcal{L}=\mathcal{L}_{0}+\sum_{k}^{N} \mathcal{J}_{k},
$$

where operators $J_{k}$ describes different jump processes into and out of the system. Let $w_{k l}(\tau)$ denote probability density function of waiting times between a jump of type $k$ and a successive jump of type $l$. A single waiting time will be denoted as $\tau_{k l}$. The Laplace transform of $w_{k l}(\tau)$ is given by the expression 


$$
w_{k l}(s)=\int_{0}^{\infty} \mathrm{e}^{-s \tau} w_{k l}(\tau) \mathrm{d} \tau=\frac{\operatorname{Tr}\left(\mathcal{J}_{l}\left(s-\mathcal{L}_{0}\right)^{-1} \mathcal{J}_{k} \rho_{0}\right)}{\operatorname{Tr}\left(\mathcal{J}_{k} \rho_{0}\right)},
$$

where $\rho_{0}$ is the vector of the stationary state. Statistical moments of the $n$-th order of the distribution $w_{k l}(\tau)$ are given by the expression

$$
\left\langle\tau_{k l}^{n}\right\rangle=(-1)^{n}\left(\frac{\mathrm{d}^{n} w_{k l}(s)}{\mathrm{d} s^{n}}\right)_{s=0} .
$$

Now I focus on the dynamics of the upper quantum dot. Let $\tau_{\mathrm{LL}}$ denote the time between subsequent jumps from the left lead to the dot. By $\tau_{\mathrm{LR}}$ I denote the time between a jump from the left lead to the dot and a successive jump from the dot to the right lead (the electron dwell time). The time $\tau_{\text {RL }}$ during which the dot is empty is defined in an analogous way. Then I calculate a covariance of subsequent times $\tau_{\mathrm{LR}}$ and $\tau_{\mathrm{RL}}$, which is a measure of their correlation. It is defined in the following way:

$$
\left\langle\Delta \tau_{\mathrm{LR}} \Delta \tau_{\mathrm{RL}}\right\rangle=\left(\left\langle\Delta \tau_{\mathrm{LL}}^{2}\right\rangle-\left\langle\Delta \tau_{\mathrm{LR}}^{2}\right\rangle-\left\langle\Delta \tau_{\mathrm{RL}}^{2}\right\rangle\right) / 2
$$

where $\Delta \tau_{k l}=\tau_{k l}-\left\langle\tau_{k l}\right\rangle$ is a deviation about the mean, $\left\langle\Delta \tau_{k l}^{2}\right\rangle=\left\langle\tau_{k l}^{2}\right\rangle-\left\langle\tau_{k l}\right\rangle^{2}$ is a variance. Normalized crosscorrelation NCC is defined as a covariance normalized to the product of standard deviations of analyzed quantities: $\mathrm{NCC}=\left\langle\Delta \tau_{\mathrm{LR}} \Delta \tau_{\mathrm{RL}}\right\rangle / \sqrt{\left\langle\Delta \tau_{\mathrm{LR}}^{2}\right\rangle\left\langle\Delta \tau_{\mathrm{RL}}^{2}\right\rangle}$.

\section{Results}

I study the dependence of the magnitude and the sign of the cross-correlation on the tunneling rates. First, a limit is considered when dynamics of the 2 nd dot is much slower than of the 1st dot. It is equivalent to the slow modulation of the quantum dot dynamics by the environment. In such situation the sign of the cross-correlation is given by the expression

$$
\operatorname{sgn}(\mathrm{NCC})=\operatorname{sgn}\left(\left(\Gamma_{\mathrm{L}}^{U}-\Gamma_{\mathrm{L}}\right)\left(\Gamma_{\mathrm{R}}^{U}-\Gamma_{\mathrm{R}}\right)\right) .
$$

This result can be understood qualitatively in the following way. Let us assume that $\Gamma_{\mathrm{L}}^{U}>\Gamma_{\mathrm{L}}$. Fast tunneling from the left lead to the dot occurs with the higher probability when the 2 nd dot is charged. If the 2 nd dot remains charged, the probability, that tunneling to the right lead will also be fast, is increased when $\Gamma_{\mathrm{R}}^{U}>\Gamma_{\mathrm{R}}$ and decreased when $\Gamma_{\mathrm{R}}^{U}<\Gamma_{\mathrm{R}}$, which makes waiting times respectively positively or negatively correlated. a)

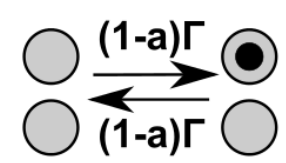

b)

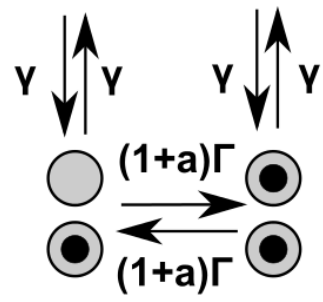

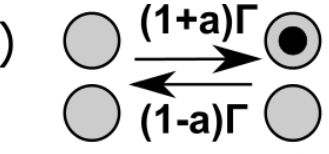

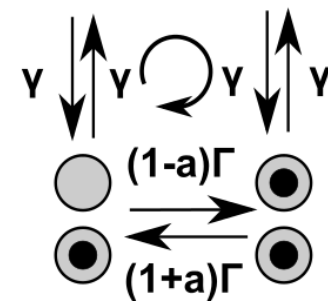

Fig. 2. Four state diagrams of the dynamics of systems with different tunneling rates symmetries. The circular arrow in (b) denotes the probability flux arising due to the broken symmetry (flowing in the clockwise direction for $a>0)$.

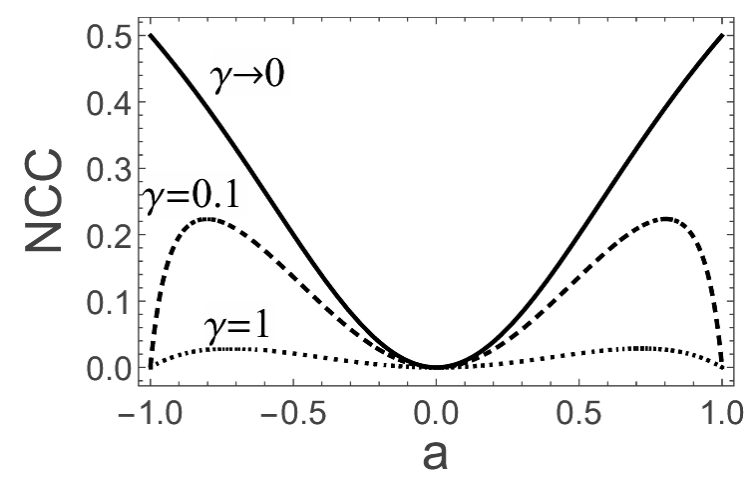

Fig. 3. Normalized cross-correlation of waiting times for the system shown in Fig. $2 \mathrm{a}$ for $\Gamma=1$.

Then I study the influence of finite values of tunneling rates between the 2nd dot and the leads on the crosscorrelation. First, I consider the system with symmetric tunneling rates: $\gamma_{i}=\gamma_{i}^{U}=\gamma, \Gamma_{i}=(1-a) \Gamma$ and $\Gamma_{i}^{U}=(1+a) \Gamma$ with $a \in[-1,1]$ (Fig. 2a). In such case value of the cross-correlation is given by the expression

$$
\mathrm{NCC}=\frac{a^{2}\left(1-a^{2}\right) \Gamma^{2}}{4 \gamma^{2}+4 \gamma \Gamma+\left(1-a^{4}\right) \Gamma^{2}} .
$$

The dependence of the cross-correlation on $a$ is shown in Fig. 3. The cross-correlation is positive in accordance with Eq. (6) and is expressed by an even function of $a$. The magnitude of the cross-correlation decreases when $\gamma$ increases. This has a following explanation: faster switching of the charge state of the 2 nd dot reduces the probability that subsequent jumps in the 1st dot will be correlated. In the limit of $\gamma \rightarrow \infty$ the correlation vanishes.

In the next step I consider the asymmetric system with $\gamma_{i}=\gamma_{i}^{U}=\gamma, \Gamma_{\mathrm{L}}=\Gamma_{\mathrm{R}}^{U}=(1+a) \Gamma$ and $\Gamma_{\mathrm{L}}^{U}=\Gamma_{\mathrm{R}}=$ $(1-a) \Gamma$ with $a \in[-1,1]$ (Fig. 2b). The dependence

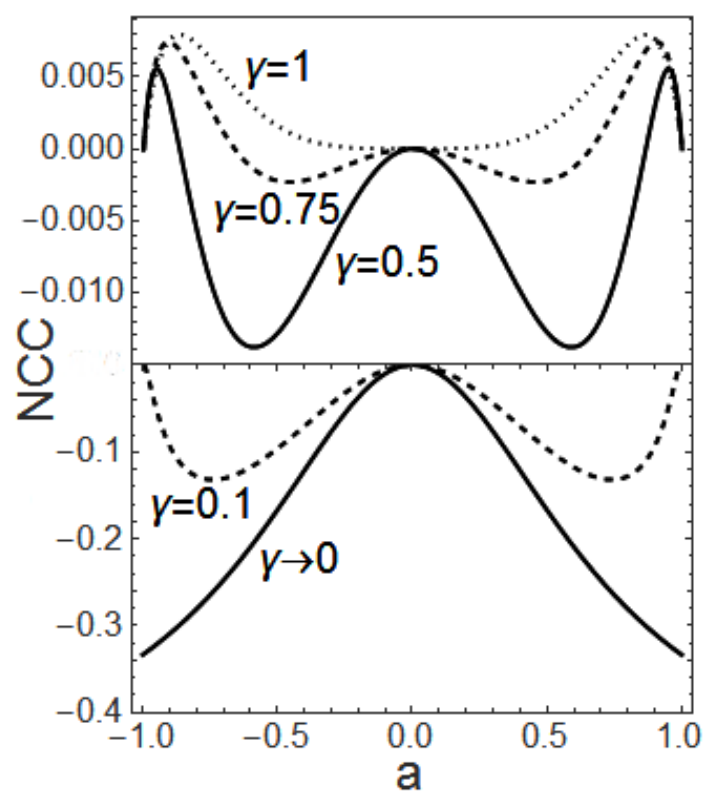

Fig. 4. Normalized cross-correlation of waiting times for the system shown in Fig. $2 \mathrm{~b}$ for $\Gamma=1$. 
of the cross-correlation on $a$ is shown in Fig. 4. In this case the cross-correlation is also expressed by an even function of $a$. For $\gamma \rightarrow 0$ the cross-correlation is always negative, in accordance with Eq. (6) and for $\gamma \rightarrow \infty$ it vanishes, as in the previously considered case. However, for finite values of $\gamma$ the cross-correlation becomes positive if $a^{2}>1-(\gamma / \Gamma)^{2}$. This can be explained using the four-state model as a result of the circular probability flux, defined as a difference of transition rates for the clockwise and the anticlockwise directions. The flux increases the probability of the following process: fast jump in the 1st dot - switching of the charge state of the 2nd dot — fast jump in the 1st dot. Thus the probability of fast transitions in 1st dot taking place subsequently is increased. If this process predominates over the subsequent jumps occurring when the charge state of the 2nd dot is fixed, cross-correlation becomes positive.

\section{Conclusions}

I have shown the correlation of waiting times between tunneling events in the capacitively interacting double quantum dot system. The magnitude and the sign of the cross-correlation can provide information about the relative change of tunneling rates due to the interaction with the second dot and about the time scale of the dynamics of the second quantum dot. The cross-correlation is especially significant, when the dynamics of one quantum dot is slowly modulated by the charge state of the other. This result suggests possible application of the waiting time correlation analysis for other cases where electronic transport is modulated by some external system, for example a spin impurity [7] or a vibrational state of a molecule [8]. Possible applications can also be find beyond the electronic transport, for example in biochemical systems, where statistical properties of the dynamics of similar Markovian models are studied [9].

\section{Acknowledgments}

I thank B.R. Bułka for the valuable discussion and comments on the manuscript. This work has been partially supported by the National Science Centre under the contract DEC-2012/05/B/ST3/03208.

\section{References}

[1] T. Brandes, Ann. Phys. (Berlin) 17, 477 (2008).

[2] S. Gustavsson, R. Leturcq, M. Studer, I. Shorubalko, T. Ihn, K. Ensslin, D.C. Driscoll, A.C. Gossard, Surf. Sci. Rep. 64, 191 (2009).

[3] D. Dasenbrock, P.P. Hofer, C. Flindt, Phys. Rev. B 91, 195420 (2015).

[4] D.T. McClure, L. DiCarlo, Y. Zhang, H.-A. Engel, C.M. Marcus, M.P. Hanson, A.C. Gossard, Phys. Rev. Lett. 98, 056801 (2007).

[5] G. Michałek, B.R. Bułka, Phys. Rev. B 80, 035320 (2009).

[6] H. Thierschmann, R. Sánchez, B. Sothmann, F. Arnold, C. Heyn, W. Hansen, H. Buhmann, L.W. Molenkamp, Nat. Nanotechnol. 10, 854 (2015).

[7] B. Sothmann, J. König, Phys. Rev. B 82, 245319 (2010).

[8] J. Koch, F. von Oppen, Phys. Rev. Lett. 94, 206804 (2005).

[9] Y.R. Chemla, J.R. Moffitt, C. Bustamante, J. Phys. Chem. B 12, 6025 (2008), A.C. Barato, U. Seifert, Phys. Rev. E 92, 032127 (2015). 